\title{
Sparfloxacin-Metal Complexes as Urease Inhibitors: Their Synthesis, Characterization, Antimicrobial, and Antienzymatic Evaluation
}

\author{
Somia Gul, ${ }^{1}$ Najma Sultana, ${ }^{2}$ M. Saeed Arayne, ${ }^{2}$ Sana Shamim, \\ Mahwish Akhtar, ${ }^{3}$ and Ajmal Khan ${ }^{4}$ \\ ${ }^{1}$ Faculty of Pharmacy, Jinnah University for Women, Karachi 74600, Pakistan \\ ${ }^{2}$ United Biotechnologies, Karach-75270, Pakistan \\ ${ }^{3}$ Dow College of Pharmacy, Dow University of Health Sciences, Karachi 74200, Pakistan \\ ${ }^{4}$ H.E.J Institute of Chemistry, University of Karachi, Karachi 75270, Pakistan \\ Correspondence should be addressed to Somia Gul; drsomi1983@yahoo.com
}

Received 29 May 2013; Revised 10 September 2013; Accepted 15 September 2013

Academic Editor: Stavros Lalas

Copyright (C) 2013 Somia Gul et al. This is an open access article distributed under the Creative Commons Attribution License, which permits unrestricted use, distribution, and reproduction in any medium, provided the original work is properly cited.

\begin{abstract}
Four new metal complexes (S12-S15) of SPFX (third-generation quinolones) via heavy metals have been synthesized in good yield and characterized by physicochemical and spectroscopic methods including TLC, IR, NMR, and elemental analyses. Sparfloxacinato ligand binds with metals through pyridone and oxygen atom of carboxylic group. The biological actives of complexes have been tested against four Gram-positive and seven Gram-negative bacteria and six different fungi. Statistical analysis of antimicrobial data was done by one-way ANOVA, Dunnett's test; it was observed that S13, S14, and S15 were found to be most active complexes. Antifungal data confirm that all four synthesized complexes are most active and show significant activity against F. solani with respect to parent drug and none of complexes show activity against A. parasiticus, A. effuris, and S. cervicis. To study inhibitory effects of newly formed complexes, enzyme inhibition studies have been conducted against urease, $\alpha$-chymotrypsin, and carbonic anhydrase. Enzymatic activity results of these complexes indicated them to be good inhibitors of urease enzyme while all complexes show mild activities against carbonic anhydrase enzyme. Further research may prove the promising role of these synthesized complexes as urease inhibitors.
\end{abstract}

\section{Introduction}

For infectious diseases, multiple therapies are usually required and so the possibility of drug-drug interactions increased. Careful consideration of concomitant drug therapy is needed. Literature survey reveals that fluoroquinolones showed several important interactions with many drugs [1]. Usually fluoroquinolones are prescribed for many diseases including respiratory and urinary tract infections. Sparfloxacin (SPFX) is an orally active synthetically broadspectrum third-generation quinolones use for upper respiratory tract infection. Metals are considered essential to a human body in performing physiologically important and vital functions, in the body [2]. The action of many drugs is dependent on coordination with metal ions or/and the inhibition on the formation of metalloenzymes [3]. The proposed mechanism of the interaction is chelation between the 4-oxo and adjacent carboxyl group of quinolone and metal cations [4-8]. Literature survey reveals that concurrent administration of magnesium and aluminium containing antacid with ciprofloxacin resulted in a nearly complete loss of activity of the drug [9] and patients who orally administrated fluoroquinolones should avoid mixtures containing multivalent cations, because quinolones binds with these metals through chelation, in consequence formed metal complex in the gastric system [10]. Ma et al. [11] published norfloxacin interaction with aluminium, magnesium and calcium and Alkaysi et al. [12] compiled interaction of 16 metals with eight quinolones. Absorption of fluoroquinolones is manifestly reduced by antacids, calcium 


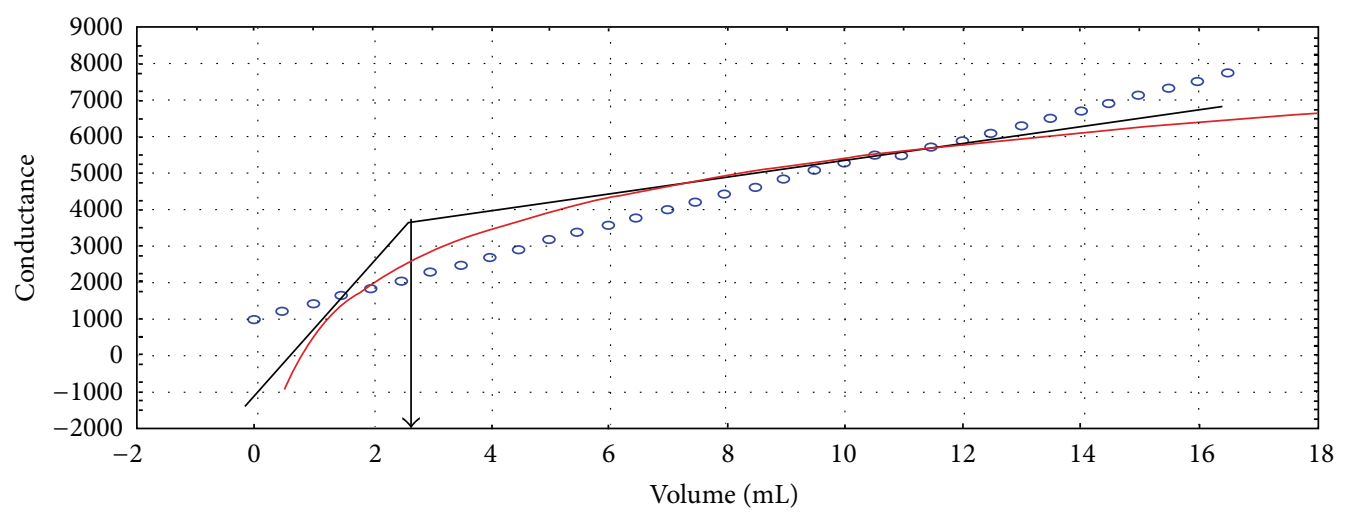

FIGURE 1: Representation of SPFX metal complexes ratio via conductance.

carbonate, ferrous sulphate, and sucralfate. Despite the fact that quantitative differences between fluoroquinolones exist, these combinations should be avoided whenever possible [7]. A reasonable recommendation may be to avoid using sucralfate and norfloxacin concurrently or avoid administration of norfloxacin and ciprofloxacin within two hours of sucralfate administration. Magnesium- and aluminum-containing antacids may also interfere with quinolones absorption. Survey assembled a number of different complexation of quinolones. Mononuclear dioxomolybdenum(VI) complexes with enrofloxacin and sparfloxacin were discovered by Efthimiadou and co-workers [13]. They also [14] discovered ciprofloxacin, cinoxacin, norfloxacin and nalidixic acid complexation with $\mathrm{VO}^{2+}, \mathrm{Mn}^{2+}, \mathrm{Fe}^{3+}, \mathrm{Co}^{2+}, \mathrm{Ni}^{2+}, \mathrm{Zn}^{2+}$, $\mathrm{MoO}^{2}, \mathrm{Cd}^{2+}$, and $\mathrm{UO}^{2+}$, vanadyl complex with enrofloxacin [15], and copper complex with sparfloxacin [16] Skyrianou et al. [17] reported nickel complex with sparfloxacin. Ciprofloxacin interaction with $\mathrm{Mn}^{2+}, \mathrm{Fe}^{3+}, \mathrm{Co}^{2+}, \mathrm{Ni}^{2+}$, and $\mathrm{MoO}^{2}$ was presented by Psomas [18]. Alkaysi et al. [19] published norfloxacin interaction with aluminum, magnesium, and calcium and Turel [20] compiled interaction of 16 metals with eight quinolones. He also published ciprofloxacin complex with $\mathrm{Cu}$ (II) [21] and Ionic complexes of protonated norfloxacin with $\mathrm{Zn}$ (II) and $\mathrm{Cu}(\mathrm{II})$ [22]. Wallis and co-workers have reported complexes of ciprofloxacin with $\mathrm{V}(\mathrm{IV}) \mathrm{O}^{2+}, \mathrm{Fe}(\mathrm{III})$ [23], and copper(II) [24]. Complexes of norfloxacin with $\mathrm{Zn}$ (II) and $\mathrm{Cu}$ (II) were prepared by Chen et al. [25] and complexes of ofloxacin with $\mathrm{Cu}$ (II) was discovered by Macías et al. [26], while Wang et al. [27] reported norfloxacin complex with Mn in 2002.

Interaction studies of SPFX metal complexes urged an idea of their synthesis [28]. Now, here we present synthesis of these complexes to aid in proving interaction studies. My research group has worked on this clinically important field of metal interaction and complexation for the last few years $[29,30]$. We have already published metal complexes of SPFX as antifungal agents [9].

In this section, spectroscopic characterization of these novel neutral mononuclear metal complexes has been conducted with spectroscopic techniques such as IR, ${ }^{1} \mathrm{H}-\mathrm{NMR}$, and elemental analyses (CHN). Prior to synthesis, $\mathrm{M}$ : $\mathrm{L}$ ratios were determined by conductance. The antimicrobial activity
TABle 1: Physicochemical parameters of SPFX and SPFX-metal complexes.

\begin{tabular}{lcccc}
\hline S. no. & Complexes & Colour & M.P ${ }^{\circ} \mathrm{C}$ & \%Yield \\
\hline 1 & SPFX & Bright yellow & 260 & - \\
2 & S12 & Yellow & 170 & 45.43 \\
3 & S13 & Light yellow & 242 & 42 \\
4 & S14 & Yellow & $238-241$ & 46 \\
5 & S15 & Dark yellow & 220 & 59.2 \\
\hline
\end{tabular}

of these complexes has been evaluated against four Grampositive and seven Gram-negative bacteria while antifungal activity against six different fungi has been determined also. Statistical analysis of antimicrobial data was done by oneway ANOVA, Dunnett's test. Enzyme inhibition studies have been conducted against urease, carbonic anhydrase, and $\alpha$-chymotrypsin enzymes. Also physiochemical parameters have been recorded carefully.

\section{Experimental}

2.1. Materials and Reagents. Sparfloxacin was a kind gift by Abbott Pharmaceuticals (Karachi) while solvents and chemicals of analytical grade were purchased from the market. Metal salts $\left(\mathrm{Al}(\mathrm{OH})_{3}, \mathrm{As}_{2} \mathrm{O}_{3}, \mathrm{AgCl}\right.$, and $\left.\mathrm{PbCO}_{3}\right)$ were of pious grade from $\mathrm{E}$. Merck. All solutions were prepared fresh before work.

2.2. Instruments. The melting points were taken on an electrothermal melting point apparatus (Gallenkamp) in open capillary tubes and are uncorrected. TLC spots were detected by UV lamp. Infrared spectra were recorded as $\mathrm{KBr}$ pellets on Shimadzu 470 instrument. ${ }^{1} \mathrm{H}$ NMR spectra were obtained by using Bruker/XWIN NMR spectrometer with TMS as internal standard. Complexes were dissolved in $\mathrm{CDCl}_{3}, \mathrm{D}_{2} \mathrm{O}$, or $\mathrm{MeOD}$ for NMR. An elemental analysis is done by Carlo Erba Strumentazione Elemental analyzer-MOD 1106 instrument.

2.3. Stoichiometric Study. Conductometric titration was performed to inspect the stoichiometric ratio of the ligand and 
TABLE 2: FTIR absorption data of SPFX and its metal complexes $\left(4000-400 \mathrm{~cm}^{-1}\right)$.

\begin{tabular}{|c|c|c|c|c|c|c|c|}
\hline S. no. & Complexes & $\mathrm{CH}-\mathrm{F}$ & Pri.NH & $\mathrm{O}-\mathrm{H}$ stretching & $\mathrm{C}=\mathrm{O}_{\mathrm{as}}$, & $\mathrm{C}=\mathrm{O}_{\mathrm{s}}$ & $\Delta^{\mathrm{a}}$ \\
\hline 1 & SPFX & 1029 & 1441 & $3346-3468^{c}$ & $1720^{\mathrm{b}}$ & - & - \\
\hline 2 & $\mathrm{~S} 12$ & 1028 & 1442 & 3336 & 1636 & 1387 & 249 \\
\hline 3 & S13 & 1027 & 1440 & 3340 & 1641 & 1387 & 254 \\
\hline 4 & S14 & 1029 & 1442 & 3347 & 1636 & 1373 & 263 \\
\hline 5 & S15 & 1027 & 1437 & 3094 & 1639 & 1368 & 271 \\
\hline
\end{tabular}

TABLE 3: ${ }^{1} \mathrm{H}-\mathrm{NMR}$ data of SPFX and its metal complexes.

\begin{tabular}{|c|c|c|}
\hline S. no. & Complexes & H-NMR $\delta:$ ppm \\
\hline 1 & SPFX & $\begin{array}{l}\text { 0.53-0.28 (3H-cyclopropyl), 3.56-3.31 (m, } 4 \mathrm{H} \text {, piperazinyl ring protons), } 4.0\left(\mathrm{NH}_{2}\right), 7.96(1 \mathrm{H} \text {-phenyl), } 8.51 \\
\text { (1H-quinolone), } 11 \text { (1H-OH, carbonyl). }\end{array}$ \\
\hline 2 & S12 & $\begin{array}{l}\text { 1.17-1.53 (5H-cyclopropyl), 3.04-3.31 (singlet piperazinyl ring), } 3.91\left(\mathrm{NH}_{2}\right), 7.24 \text { (1H-phenyl), } 8.62 \\
\text { (1H-quinolone). }\end{array}$ \\
\hline 3 & S13 & $\begin{array}{l}\text { 1.04-1.20 (5H-cyclopropyl), 3.29-3.31 (singlet piperazinyl ring), } 3.92\left(\mathrm{NH}_{2}\right), 6.44 \text { (1H-phenyl), } 8.62 \\
\text { (1H-quinolone). }\end{array}$ \\
\hline 4 & S14 & $1.03-1.06$ (3H-cyclopropyl), 3.20-3.31 (piperzinyl), $3.98\left(\mathrm{NH}_{2}\right), 6.450-7.20$ (phenyl), $8.46\left(1 \mathrm{H}-\mathrm{CH}_{2}\right)$. \\
\hline 5 & S15 & $\begin{array}{l}\text { 1.17-1.21 (4H-cyclopropyl), 3.29-3.31 (singlet piperazinyl ring), } 3.90\left(\mathrm{NH}_{2}\right), 6.44 \text { (1H-phenyl), } 8.61 \\
\text { (1H-quinolone). }\end{array}$ \\
\hline
\end{tabular}

TABLE 4: Elemental analyses of the complexes.

\begin{tabular}{lccc}
\hline Metal complexes & Found (calcd.) $\%$ & Found (calcd.) \% & Found (calcd.) \% \\
\hline SPFX & C & $5.65(5.53)$ & N \\
{$\left[\mathrm{Al}(\mathrm{SPFX})_{2}\right] \cdot 3 \mathrm{H}_{2} \mathrm{O}$} & $58.16(57.09)$ & $5.96(5.78)$ & $14.28(14.06)$ \\
{$\left[\mathrm{As}(\mathrm{SPFX})_{2}\right] \cdot 2 \mathrm{H}_{2} \mathrm{O}$} & $53.24(53.76)$ & $5.76(5.48)$ & $12.74(12.52)$ \\
{$\left[\mathrm{Ag}(\mathrm{SPFX})_{2}\left(\mathrm{H}_{2} \mathrm{O}\right)_{2}\right] \mathrm{Cl}_{2} \cdot 2 \mathrm{H}_{2} \mathrm{O}$} & $53.55(53.63)$ & $4.92(5.23)$ & $12.81(12.75)$ \\
{$\left[\mathrm{Pb}(\mathrm{SPFX})_{2} \cdot\left(\mathrm{H}_{2} \mathrm{O}\right)_{2}\right] \mathrm{CO}_{3}$} & $49.72(49.66)$ & $4.35(4.42)$ & $11.89(11.82)$ \\
\hline
\end{tabular}

metal ions. For this purpose, $1 \mathrm{mM}$ alcoholic solutions of drug (SPFX) and metal salts were prepared individually. In $20 \mathrm{~mL}$ of drug (SPFX) solution, $2 \mathrm{~mL}$ of metal solution was added each time; after every 2 min the conductance value was carefully noted. All the values of conductance were noted until state of chemical equilibrium is achieved. Graph was plotted between corrected conductivity and the volume of titrant added and the end point was determined. Results show that all complexes have stoichiometries of $2: 1$ (drug: metal). Figure 1 represents conductometric ratio.

2.4. Synthesis of Complexes. A warm methanolic, unimolar solution of metal salts was mixed with a bimolar solution of SPFX in methanol $(1: 2)$ in round bottomed flask and was refluxed for $4 \mathrm{~h}$, above $80^{\circ} \mathrm{C}$ on a water bath with constant stirring. The solution was filtered and the product left for slow evaporation and then crystallized at room temperature. After a few days, crystals deposited were collected, washed with methanol, and dried. \% yield, color, melting points, and solubility of all the complexes were carried out in different solvents as water, methanol, chloroform, and dimethyl sulfoxide.

2.5. Antimicrobial Activity. For antibacterial and antifungal studies, disk susceptibility technique was used. The diffusion technique developed by Bauer et al. [31] recommended by the FDA [32] has been adopted which has most extensively been used in the clinical laboratories [33].

2.6. Preparation of Dried Paper Disk. The stock solutions of standard drug (SPFX) and SPFX-metal complexes were prepared in water to get the concentration of $100 \mu \mathrm{gmL}^{-1}$ and diluted in four concentrations of $40,20,10$, and $5 \mu \mathrm{gmL}^{-1}$. Three $\mathrm{mm}$ filter paper discs were impregnated with $20 \mathrm{~mL}$ of each of the different dilutions.

Discs were allowed to remain at room temperature till complete diluents evaporation and kept under refrigeration (ready to be used).

2.7. Procedure for Antimicrobial Activity. Organisms studied were taken from the slant with the help of wire loop and were immersed in the tube containing nutrient broth which was incubated at $37^{\circ} \mathrm{C}$ for $4-6 \mathrm{hrs}$ until the turbidity exceeded that of $0.5 \mathrm{MacFarland}$ standards. Nutrient agar was prepared, autoclaved at $121^{\circ} \mathrm{C}$ for 15 , minutes then poured in dry, sterile Petri dishes, cooled, and set. The bacterial inoculum was uniformly spread using sterile cotton swab on a sterile Petri dish with agar. Discs soaked with metal complexes and derivatives were placed onto the surface of the agar with bacterial inoculum and sparfloxacin disk was used for control. These were then incubated at $36^{\circ} \mathrm{C} \pm 1^{\circ} \mathrm{C}$, for $24 \mathrm{~h}$, 

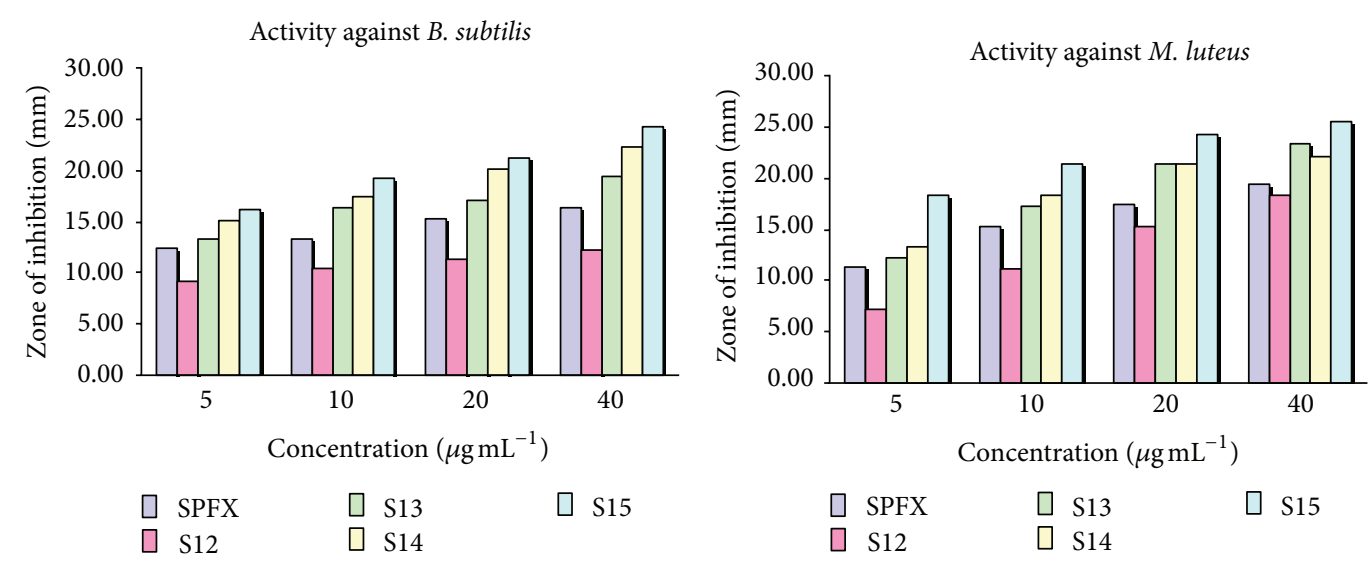
$\square$ SPFX
$\square \mathrm{S} 13$
口S15
S 12
․ S14
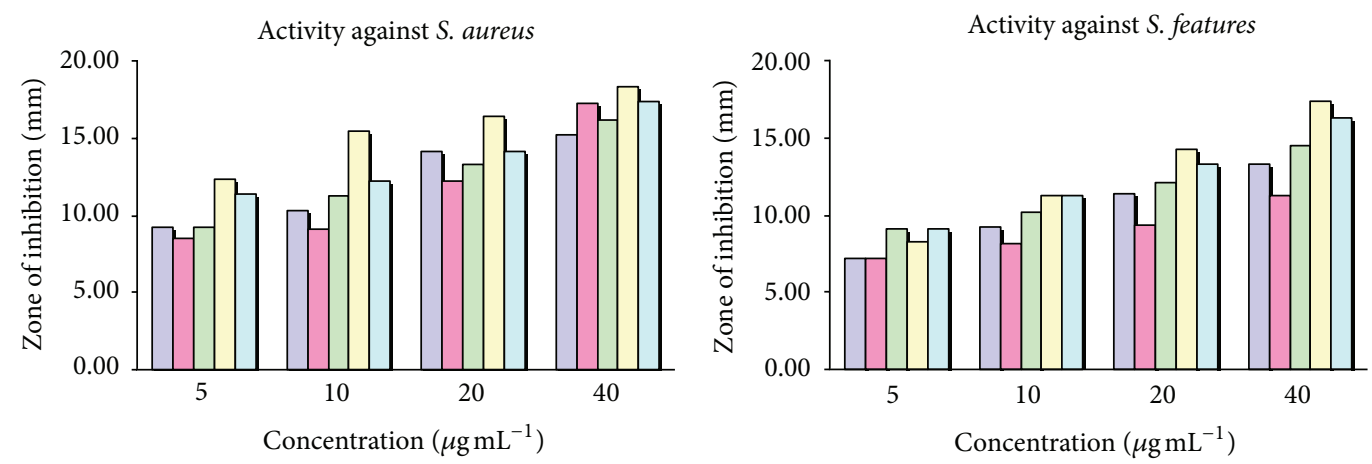

$\begin{array}{lll}\square \text { SPFX } & \square \text { S13 } & \square \text { S15 } \\ \square \text { S12 } & \square \text { S14 } & \end{array}$

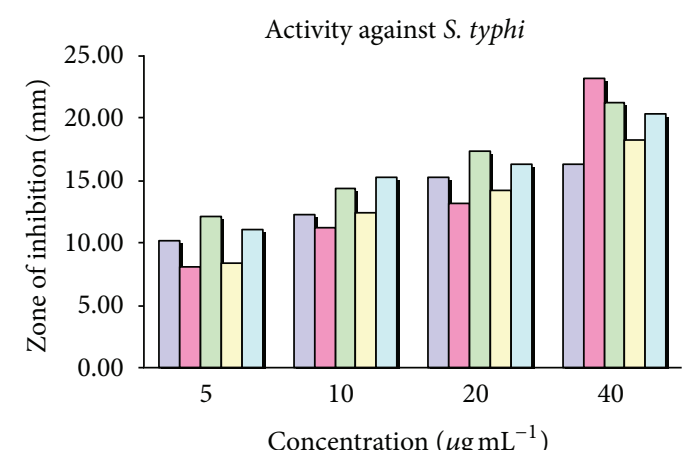

$\begin{array}{lll}\square \text { SPFX } & \square & \square\end{array}$

$\begin{array}{ll}\text { S12 } & \text { S14 }\end{array}$

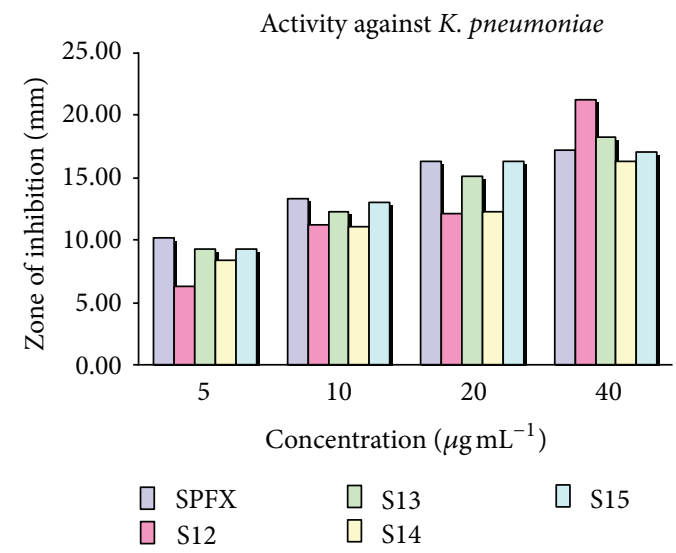

$\begin{array}{lll}\square \text { SPFX } & \square \text { S13 } & \square \text { S15 } \\ \square \text { S12 } & \square \text { S14 } & \end{array}$

$\begin{array}{ll}\square \text { S12 } & \square \text { S14 }\end{array}$
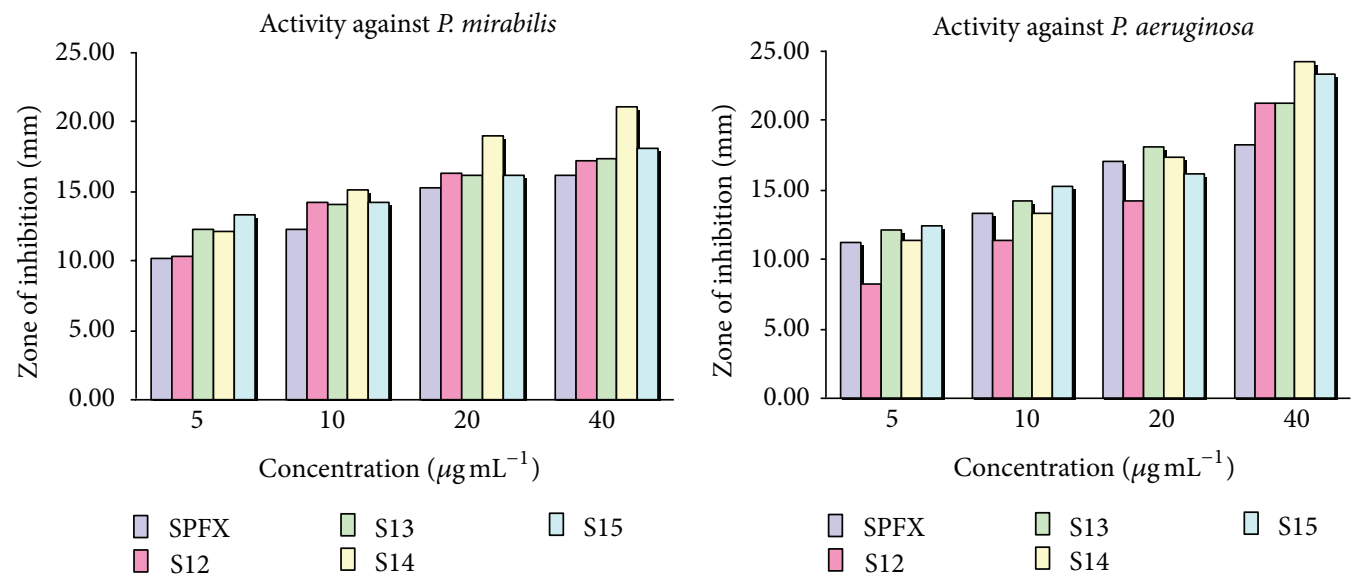

(a)

FIgURe 2: Continued. 

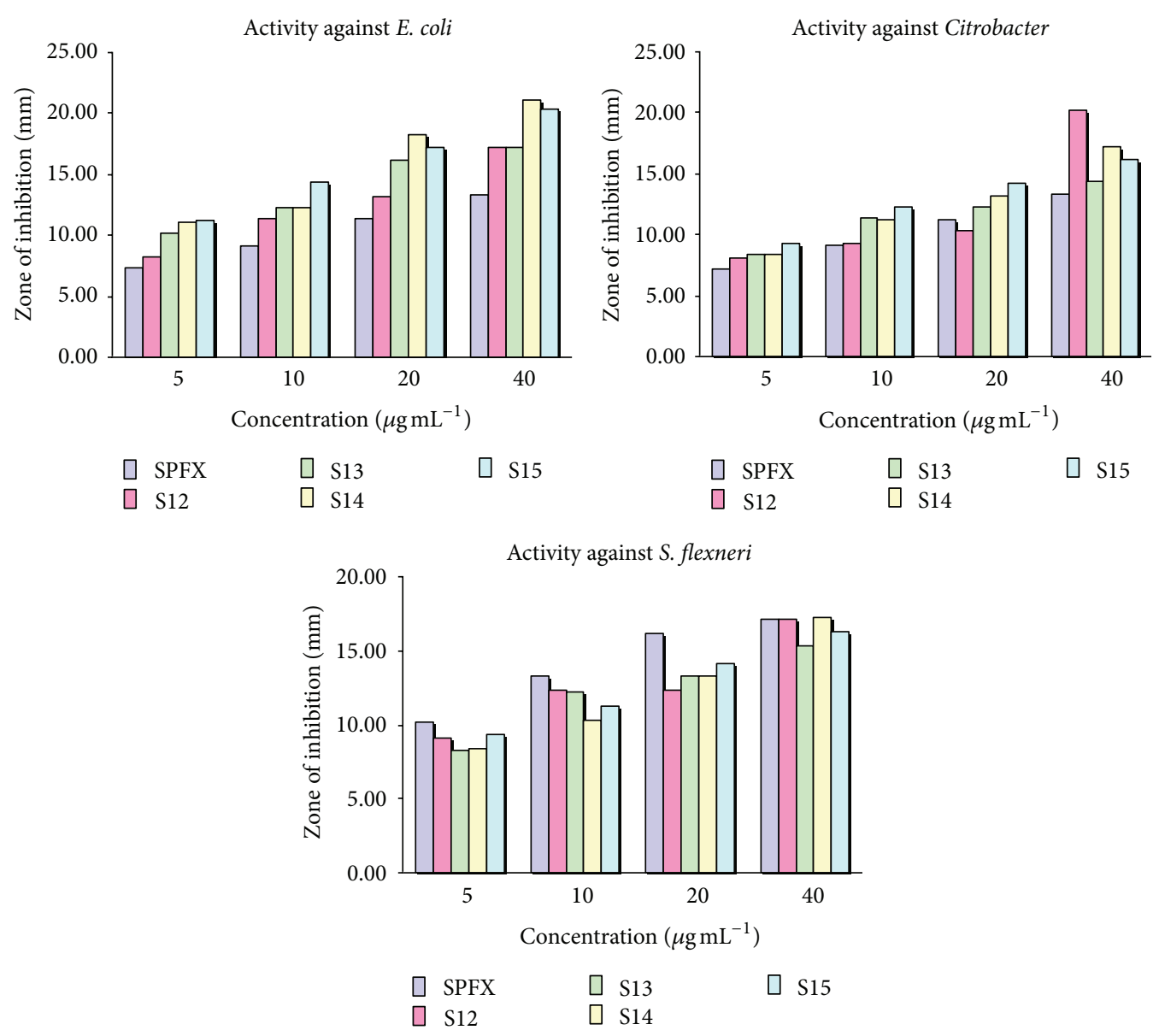

(b)

FIGURE 2: Graphical representation of inhibition zone against Gram-positive and Gram-negative bacteria.

while the water paper discs were used as a positive control. Three replicate trials were conducted against each organism for each concentration. Statistical analysis was used for data interpretation included calculation of the mean values, standard deviation, and investigation of significant differences in results. Similar procedures were adopted for antifungal activities. Derivative discs $\left(5,10,20\right.$, and $\left.40 \mu \mathrm{gmL}^{-1}\right)$ were placed on SDS medium plates previously seeded with fungal culture and incubated for seven days at $36^{\circ} \mathrm{C} \pm 1^{\circ} \mathrm{C}$, for 48 hours. Zones of inhibition were carefully measured using Vernier caliper.

2.8. Statistical Study. Statistical analysis of antimicrobial data was done by one-way ANOVA, Dunnett's test through SPSS software version 10.0 (Carry, NC, USA).

\section{Results and Discussion}

3.1. Synthesis of SPFX-Metal Complexes with Heavy Metals. Four metal complexes were synthesized by refluxing metal salt solutions of $\mathrm{Al}(\mathrm{OH})_{3}, \mathrm{As}_{2} \mathrm{O}_{3}, \mathrm{AgCl}$, and $\mathrm{Pb}_{2} \mathrm{CO}_{3}$ in methanol with SPFX in the ratio of $1: 2[\mathrm{M}: \mathrm{L}]$ (determined by conductance), for 4 hours, and the volume was reduced by evaporation. Moreover, their melting points and solubility were noted. Solubility facts of these complexes show that $\mathrm{Al}^{3+}$ and $\mathrm{As}^{3+}$ were soluble in $\mathrm{CdCl} 3, \mathrm{Agl}+$ was soluble in $\mathrm{MeOH}$, and $\mathrm{Pb}^{3+}$ was soluble in both $\mathrm{MeOH}$ and $\mathrm{CdCl} 3$. Physicochemical parameters of SPFX and SPFX-metal complexes are given in Table 1 . The antimicrobial activity of these complexes has been evaluated against mentioned bacteria and fungi and analysis of data was done by one-way ANOVA. Enzyme inhibition studies have been conducted against the abovementioned enzymes.

3.2. Proposed Structure of SPFX Metal Complexes. The coordination chemistry of some quinolones (including sparfloxacin) antibiotics with transition and d10 metal ions has been reported [34-37]. In this case, the SPFX has several potential donor sites but, due to steric hindrances, the ligand can provide a maximum of two donor atoms to any one metal centre. The spectroscopic changes suggested that the SPFX acts as a bidentate ligand and its coordination occurs through the metal via the pyridone and one carboxylato oxygen atom and forms slightly distorted octahedral geometry. 


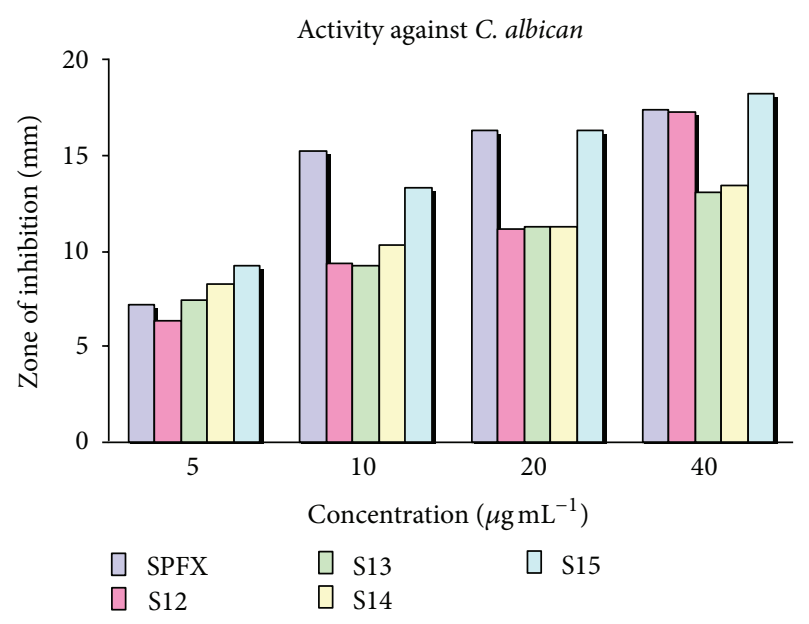

(a)

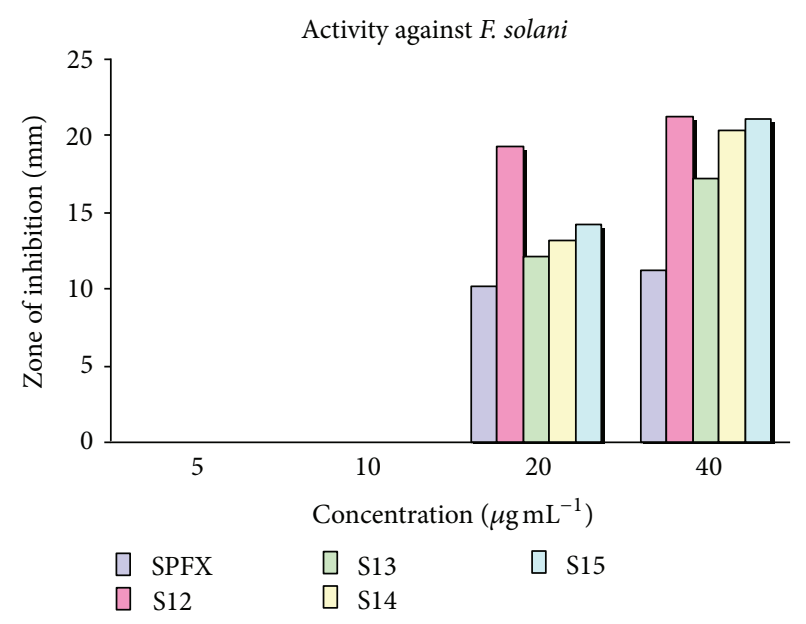

(b)

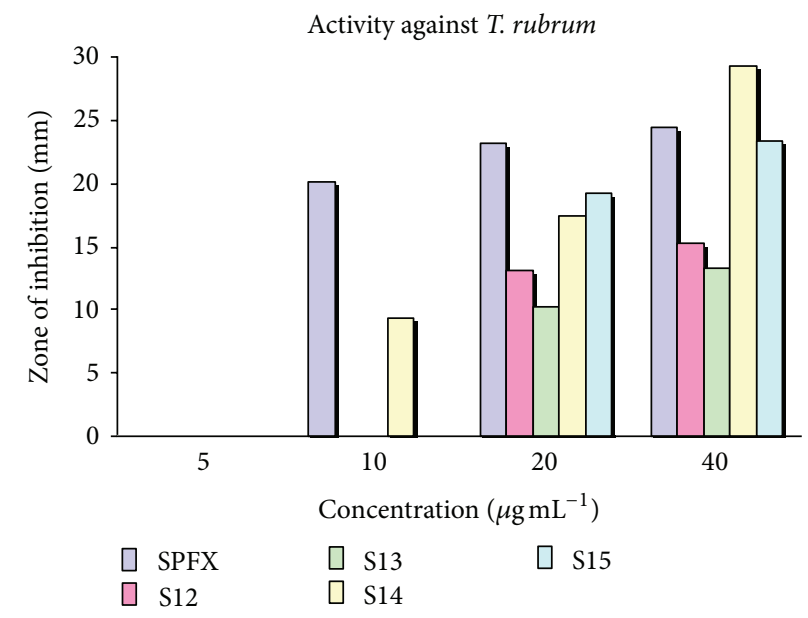

(c)

FIGURE 3: Graphical representation of inhibition zone against fungi.

\subsection{Spectroscopic Studies}

3.3.1. Infrared Analysis. IR spectra of SPFX_M.complex (S12S15) revealed that the absorption at $1720 \mathrm{~cm}^{-1}$ observed in the spectrum of sparfloxacin, attributed to the $v(\mathrm{C}=\mathrm{O})_{\text {carb }}$, has been replaced with two very strong characteristic bands in the range of $1636-1641 \mathrm{~cm}^{-1}$ and $1368-1387 \mathrm{~cm}^{-1}$ assigned as $v(\mathrm{O}-\mathrm{C}-\mathrm{O})$ asymmetric and symmetric stretching vibrations, respectively (Table 2) [31]. The $\Delta\left[v_{\text {asym }}\left(\mathrm{CO}_{2}\right)-v_{\text {sym }}\left(\mathrm{CO}_{2}\right)\right]$ values fall in the range of $249-271 \mathrm{~cm}^{-1}$ indicating a monodentate coordination mode of the carboxylato group via the pyridone and one carboxylato oxygen atom [7]. The vibration $v(\mathrm{C}=\mathrm{O})_{\mathrm{p}}$, pyridone stretch is slightly shifted from 1641 to $1636-1641 \mathrm{~cm}^{-1}$ upon bonding [35]. Broad split band at 3094$3347 \mathrm{~cm}^{-1}$ can be assigned to the $\mathrm{O}-\mathrm{H}$ stretching vibrations of water molecules and also includes the $\mathrm{N}-\mathrm{H}$ stretching vibration of the piperazinyl moiety [38, 39]. New bands around $470-490 \mathrm{~cm}^{-1}$ seemed in the spectra of complexes can be assigned to $v(\mathrm{M}-\mathrm{O})[40]$.
3.3.2. ${ }^{1} H$ NMR Analysis. The proton NMR spectrum of complexes showed a set of signals which were almost identical to those of SPFX, while changes occurred particularly at carboxylic protons as well as protons of aromatic C-NH. Singlet at $\delta 3.90-3.98 \mathrm{ppm}$ was assigned to $\mathrm{C}-\mathrm{NH}_{2}$ group. The spectra showed multiplet at $\delta 1.03-1.53$ ppm for cyclopropyl protons, multiplet at $\delta 3.04-3.31 \mathrm{ppm}$ for iperazinyl protons, and singlet at $\delta 8.46-8.62 \mathrm{ppm}$ for $-\mathrm{CH}_{2}$ protons (Table 3 ). No broad weak band for acidic proton at $\delta: 11 \mathrm{ppm}$ seen in spectra of complexes indicating that this targeted moiety took part in complexation of metals with SPFX and SPFX acts as bidentate deprotonated ligands bound to the metal through the pyridone oxygen and one carboxylate oxygen [9].

3.3.3. Elemental Analysis. The results obtained from elemental analysis CHN point toward that all of the complexes are formed from the reaction of the metal salt with drug in $1: 2$ molar ratio (Table 4). 
TABLE 5: (a) Inhibition zones (mm) against Bacillus subtilis. (b) Inhibition zones (mm) against Micrococcus luteus. (c) Inhibition zones (mm) against Staphylococcus aureus. (d) Inhibition zones ( $\mathrm{mm}$ ) against Streptococcus features. (e) Inhibition zones (mm) against Salmonella typhi. (f) Inhibition zones ( $\mathrm{mm}$ ) against Klebsiella pneumonia. (g) Inhibition zones (mm) against Proteus mirabilis. (h) Inhibition zones (mm) against Pseudomonas aeruginosa. (i) Inhibition zones ( $\mathrm{mm}$ ) against Escherichia coli. (j) Inhibition zones (mm) against Citrobacter species. (k) Inhibition zones $(\mathrm{mm})$ against Shigella flexneri.

(a)

\begin{tabular}{lcccr}
\hline \multirow{2}{*}{ Complexes } & \multicolumn{4}{c}{ Concentrations $\left(\mu \mathrm{gmL}^{-1}\right)$} \\
& $5\left(\mu \mathrm{gmL}^{-1}\right)$ & $10\left(\mu \mathrm{gmL}^{-1}\right)$ & $20\left(\mu \mathrm{gmL}^{-1}\right)$ & $40(\mu \mathrm{gmL})$ \\
\hline SPFX & $7.21 \pm 0.2$ & $15.3 \pm 0.21$ & $16.31 \pm 0.04$ & $17.28 \pm 0.11$ \\
S12 & $8.09 \pm 0.36^{* *}(-12.21)$ & $9.29 \pm 0.1^{* *}(39.28)$ & $11.1 \pm 0.08^{* *}(31.94)$ & $14.05 \pm 0.25^{* *}(18.69)$ \\
S13 & $8.84 \pm 0.16^{* *}(-22.61)$ & $11.01 \pm 0.27^{* *}(28.04)$ & $15.08 \pm 0.18^{* *}(7.54)$ & $18.32 \pm 0.14^{* *}(-6.02)$ \\
S14 & $0 \pm 0^{* *}(100)$ & $0 \pm 0^{* *}(100)$ & $9.31 \pm 0.25^{* *}(42.92)$ & $10.28 \pm 0.23^{* *}(40.51)$ \\
S15 & $6.97 \pm 0.07^{* *}(3.33)$ & $10.16 \pm 0.2^{* *}(33.59)$ & $12.21 \pm 0.12^{* *}(25.14)$ & $14.37 \pm 0.06^{* *}(16.84)$ \\
ANOVA $(\mathrm{df}=10,22)$ & $\mathrm{F}-334.993 P<0.001$ & $\mathrm{~F}-44.427 P<0.001$ & $\mathrm{~F}-132.338 P<0.001$ & $\mathrm{~F}-123.338 P<0.001$ \\
\hline
\end{tabular}

Mean \pm S.D (percent zone of inhibition), ${ }^{* *} P<0.005,{ }^{*} P<0.05$.

(b)

\begin{tabular}{lcccc}
\hline \multirow{2}{*}{ Complexes } & \multicolumn{3}{c}{ Concentrations $\left(\mu \mathrm{gmL}^{-1}\right)$} \\
& $5\left(\mu \mathrm{gmL}^{-1}\right)$ & $10\left(\mu \mathrm{gmL}^{-1}\right)$ & $20\left(\mu \mathrm{gmL}^{-1}\right)$ & $40\left(\mu \mathrm{gmL}^{-1}\right)$ \\
\hline SPFX & $7.21 \pm 0.2$ & $15.3 \pm 0.21$ & $16.31 \pm 0.04$ & $17.28 \pm 0.11$ \\
S12 & $8.09 \pm 0.36^{* *}(-12.21)$ & $9.29 \pm 0.1^{* *}(39.28)$ & $11.1 \pm 0.08^{* *}(31.94)$ & $14.05 \pm 0.25^{* *}(18.69)$ \\
S13 & $8.84 \pm 0.16^{* *}(-22.61)$ & $11.01 \pm 0.27^{* *}(28.04)$ & $15.08 \pm 0.18^{* *}(7.54)$ & $18.32 \pm 0.14^{* *}(-6.02)$ \\
S14 & $0 \pm 0^{* *}(100)$ & $0 \pm 0^{* *}(100)$ & $9.31 \pm 0.25^{* *}(42.92)$ & $10.28 \pm 0.23^{* *}(40.51)$ \\
S15 & $6.97 \pm 0.07^{* *}(3.33)$ & $10.16 \pm 0.2^{* *}(33.59)$ & $12.21 \pm 0.12^{* *}(25.14)$ & $14.37 \pm 0.06^{* *}(16.84)$ \\
ANOVA $(\mathrm{df}=10,22)$ & $\mathrm{F}-742.153 P<0.001$ & $\mathrm{~F}-192.257 P<0.001$ & $\mathrm{~F}-760.801 P<0.001$ & $\mathrm{~F}-467.658 P<0.001$ \\
\hline
\end{tabular}

Mean \pm S.D (percent zone of inhibition), ${ }^{* *} P<0.005,{ }^{*} P<0.05$.

(c)

\begin{tabular}{lcccc}
\hline Complexes & \multicolumn{4}{c}{ Concentrations $\left(\mu \mathrm{gmL}^{-1}\right)$} \\
& 5 & 10 & 16 & 40 \\
\hline SPFX & $7.21 \pm 0.2$ & $15.3 \pm 0.21$ & $16.31 \pm 0.04$ & $17.28 \pm 0.11$ \\
S12 & $8.09 \pm 0.36^{* *}(-12.21)$ & $9.29 \pm 0.1^{* *}(39.28)$ & $11.1 \pm 0.08^{* *}(31.94)$ & $14.05 \pm 0.25^{* *}(18.69)$ \\
S13 & $8.84 \pm 0.16^{* *}(-22.61)$ & $11.01 \pm 0.27^{* *}(28.04)$ & $15.08 \pm 0.18^{* *}(7.54)$ & $18.32 \pm 0.14^{* *}(-6.02)$ \\
S14 & $0 \pm 0^{* *}(100)$ & $0 \pm 0^{* *}(100)$ & $9.31 \pm 0.25^{* *}(42.92)$ & $10.28 \pm 0.23^{* *}(40.51)$ \\
S15 & $6.97 \pm 0.07^{* *}(3.33)$ & $10.16 \pm 0.2^{* *}(33.59)$ & $12.21 \pm 0.12^{* *}(25.14)$ & $14.37 \pm 0.06^{* *}(16.84)$ \\
ANOVA $(\mathrm{df}=10,22)$ & $\mathrm{F}-434.618 P<0.001$ & $\mathrm{~F}-200.157 P<0.001$ & $\mathrm{~F}-680.164 P<0.001$ & $\mathrm{~F}-326.531 P<0.001$ \\
\hline
\end{tabular}

Mean \pm S.D (percent zone of inhibition), ${ }^{* *} P<0.005,{ }^{*} P<0.05$.

(d)

\begin{tabular}{lcccc}
\hline Complexes & 5 & \multicolumn{3}{c}{ Concentrations $\left(\mu \mathrm{gmL}^{-1}\right)$} \\
& 5 & 10 & $16.31 \pm 0.04$ & 40 \\
\hline SPFX & $7.21 \pm 0.2$ & $15.3 \pm 0.21$ & $11.1 \pm 0.08^{* *}(31.94)$ & $14.05 \pm 0.25^{* *}(18.69)$ \\
S12 & $8.09 \pm 0.36^{* *}(-12.21)$ & $9.29 \pm 0.1^{* *}(39.28)$ & $15.08 \pm 0.18^{* *}(7.54)$ & $18.32 \pm 0.14^{* *}(-6.02)$ \\
S13 & $8.84 \pm 0.16^{* *}(-22.61)$ & $11.01 \pm 0.27^{* *}(28.04)$ & $9.31 \pm 0.25^{* *}(42.92)$ & $10.28 \pm 0.23^{* *}(40.51)$ \\
S14 & $0 \pm 0^{* *}(100)$ & $0 \pm 0^{* *}(100)$ & $12.21 \pm 0.12^{* *}(25.14)$ & $14.37 \pm 0.06^{* *}(16.84)$ \\
S15 & $6.97 \pm 0.07^{* *}(3.33)$ & $10.16 \pm 0.2^{* *}(33.59)$ & F-622.545P<0.001 & F-420.443P<0.001 \\
ANOVA $(\mathrm{df}=10,22)$ & $\mathrm{F}-1304.177 P<0.001$ & F-1543.733P<0.001 &
\end{tabular}

Mean \pm S.D (percent zone of inhibition), ${ }^{* *} P<0.005,{ }^{*} P<0.05$.

(e)

\begin{tabular}{lcccc}
\hline \multirow{2}{*}{ Complexes } & \multicolumn{4}{c}{ Concentrations $\left(\mu \mathrm{gmL}^{-1}\right)$} \\
& 5 & 10 & 20 & 40 \\
\hline SPFX & $7.21 \pm 0.2$ & $15.3 \pm 0.21$ & $16.31 \pm 0.04$ & $17.28 \pm 0.11$ \\
\hline
\end{tabular}


(e) Continued.

\begin{tabular}{lcccc}
\hline Complexes & 5 & \multicolumn{4}{c}{ Concentrations $\left(\mu \mathrm{gmL}^{-1}\right)$} \\
& 5 & 10 & 20 & 40 \\
\hline S12 & $8.09 \pm 0.36^{* *}(-12.21)$ & $9.29 \pm 0.1^{* *}(39.28)$ & $11.1 \pm 0.08^{* *}(31.94)$ & $14.05 \pm 0.25^{* *}(18.69)$ \\
S13 & $8.84 \pm 0.16^{* *}(-22.61)$ & $11.01 \pm 0.27^{* *}(28.04)$ & $15.08 \pm 0.18^{* *}(7.54)$ & $18.32 \pm 0.14^{* *}(-6.02)$ \\
S14 & $0 \pm 0^{* *}(100)$ & $0 \pm 0^{* *}(100)$ & $9.31 \pm 0.25^{* *}(42.92)$ & $10.28 \pm 0.23^{* *}(40.51)$ \\
S15 & $6.97 \pm 0.07^{* *}(3.33)$ & $10.16 \pm 0.2^{* *}(33.59)$ & $12.21 \pm 0.12^{* *}(25.14)$ & $14.37 \pm 0.06^{* *}(16.84)$ \\
ANOVA $(\mathrm{df}=10,22)$ & $\mathrm{F}-1304.177 P<0.001$ & $\mathrm{~F}-1543.733 P<0.001$ & $\mathrm{~F}-622.545 P<0.001$ & $\mathrm{~F}-420.443 P<0.001$ \\
\hline
\end{tabular}

Mean \pm S.D (percent zone of inhibition), ${ }^{* *} P<0.005,{ }^{*} P<0.05$.

(f)

\begin{tabular}{|c|c|c|c|c|}
\hline \multirow{2}{*}{ Complexes } & \multicolumn{4}{|c|}{ Concentrations $\left(\mu \mathrm{gmL}^{-1}\right)$} \\
\hline & 5 & 10 & 20 & 40 \\
\hline SPFX & $7.21 \pm 0.2$ & $15.3 \pm 0.21$ & $16.31 \pm 0.04$ & $17.28 \pm 0.11$ \\
\hline $\mathrm{S} 12$ & $8.09 \pm 0.36^{* *}(-12.21)$ & $9.29 \pm 0.1^{* *}(39.28)$ & $11.1 \pm 0.08^{* *}(31.94)$ & $14.05 \pm 0.25^{* *}(18.69)$ \\
\hline S13 & $8.84 \pm 0.16^{* *}(-22.61)$ & $11.01 \pm 0.27^{* *}(28.04)$ & $15.08 \pm 0.18^{* *}(7.54)$ & $18.32 \pm 0.14^{* *}(-6.02)$ \\
\hline S14 & $0 \pm 0^{* *}(100)$ & $0 \pm 0^{* *}(100)$ & $9.31 \pm 0.25^{* *}(42.92)$ & $10.28 \pm 0.23^{* *}(40.51)$ \\
\hline S15 & $6.97 \pm 0.07^{* *}(3.33)$ & $10.16 \pm 0.2^{* *}(33.59)$ & $12.21 \pm 0.12^{* *}(25.14)$ & $14.37 \pm 0.06^{* *}(16.84)$ \\
\hline $\operatorname{ANOVA}(\mathrm{df}=10,22)$ & F-1304.177 $P<0.001$ & F-1543.733 $P<0.001$ & F-622.545 $P<0.001$ & F-420.443 $P<0.001$ \\
\hline
\end{tabular}

Mean \pm S.D (percent zone of inhibition), ${ }^{* *} P<0.005,{ }^{*} P<0.05$.

(g)

\begin{tabular}{|c|c|c|c|c|}
\hline \multirow{2}{*}{ Complexes } & \multicolumn{4}{|c|}{ Concentrations $\left(\mu \mathrm{gmL}^{-1}\right)$} \\
\hline & 5 & 10 & 20 & 40 \\
\hline SPFX & $7.21 \pm 0.2$ & $15.3 \pm 0.21$ & $16.31 \pm 0.04$ & $17.28 \pm 0.11$ \\
\hline S12 & $8.09 \pm 0.36^{* *}(-12.21)$ & $9.29 \pm 0.1^{* *}(39.28)$ & $11.1 \pm 0.08^{* *}(31.94)$ & $14.05 \pm 0.25^{* *}(18.69)$ \\
\hline S13 & $8.84 \pm 0.16^{* *}(-22.61)$ & $11.01 \pm 0.27^{* *}(28.04)$ & $15.08 \pm 0.18^{* *}(7.54)$ & $18.32 \pm 0.14^{* *}(-6.02)$ \\
\hline S14 & $0 \pm 0^{* *}(100)$ & $0 \pm 0^{* *}(100)$ & $9.31 \pm 0.25^{* *}(42.92)$ & $10.28 \pm 0.23^{* *}(40.51)$ \\
\hline S15 & $6.97 \pm 0.07^{* *}(3.33)$ & $10.16 \pm 0.2^{* *}(33.59)$ & $12.21 \pm 0.12^{* *}(25.14)$ & $14.37 \pm 0.06^{* *}(16.84)$ \\
\hline $\operatorname{ANOVA}(\mathrm{df}=10,22)$ & F-1304.177 $P<0.001$ & F-1543.733 $P<0.001$ & F-622.545 $P<0.001$ & F-420.443 $P<0.001$ \\
\hline
\end{tabular}

Mean \pm S.D (percent zone of inhibition), ${ }^{* *} P<0.005,{ }^{*} P<0.05$.

(h)

\begin{tabular}{|c|c|c|c|c|}
\hline \multirow{2}{*}{ Complexes } & \multicolumn{4}{|c|}{ Concentrations $\left(\mu \mathrm{gmL}^{-1}\right)$} \\
\hline & 5 & 10 & 20 & 40 \\
\hline SPFX & $7.21 \pm 0.2$ & $15.3 \pm 0.21$ & $16.31 \pm 0.04$ & $17.28 \pm 0.11$ \\
\hline S12 & $8.09 \pm 0.36^{* *}(-12.21)$ & $9.29 \pm 0.1^{* *}(39.28)$ & $11.1 \pm 0.08^{* *}(31.94)$ & $14.05 \pm 0.25^{* *}(18.69)$ \\
\hline S13 & $8.84 \pm 0.16^{* *}(-22.61)$ & $11.01 \pm 0.27^{* *}(28.04)$ & $15.08 \pm 0.18^{* *}(7.54)$ & $18.32 \pm 0.14^{* *}(-6.02)$ \\
\hline S14 & $0 \pm 0^{* *}(100)$ & $0 \pm 0^{* *}(100)$ & $9.31 \pm 0.25^{* *}(42.92)$ & $10.28 \pm 0.23^{* *}(40.51)$ \\
\hline S15 & $6.97 \pm 0.07^{* *}(3.33)$ & $10.16 \pm 0.2^{* *}(33.59)$ & $12.21 \pm 0.12^{* *}(25.14)$ & $14.37 \pm 0.06^{* *}(16.84)$ \\
\hline $\operatorname{ANOVA}(\mathrm{df}=10,22)$ & F-1304.177 $P<0.001$ & F-1543.733 $P<0.001$ & F-622.545 $P<0.001$ & F-420.443 $P<0.001$ \\
\hline
\end{tabular}

Mean \pm S.D (percent zone of inhibition), ${ }^{* *} P<0.005,{ }^{*} P<0.05$.

(i)

\begin{tabular}{lccrr}
\hline Complexes & \multicolumn{4}{c}{ Concentrations $\left(\mu \mathrm{gmL}^{-1}\right)$} \\
& 5 & 10 & $16.31 \pm 0.04$ & 40 \\
\hline SPFX & $7.21 \pm 0.2$ & $15.3 \pm 0.21$ & $11.1 \pm 0.08^{* *}(31.94)$ & $17.28 \pm 0.11$ \\
S12 & $8.09 \pm 0.36^{* *}(-12.21)$ & $9.29 \pm 0.1^{* *}(39.28)$ & $15.08 \pm 0.18^{* *}(7.54)$ & $18.05 \pm 0.25^{* *}(18.69)$ \\
S13 & $8.84 \pm 0.16^{* *}(-22.61)$ & $11.01 \pm 0.27^{* *}(28.04)$ & $9.31 \pm 0.25^{* *}(42.92)$ & $10.32 \pm 0.14^{* *}(-6.02)$ \\
S14 & $0 \pm 0^{* *}(100)$ & $0 \pm 0^{* *}(100)$ & $12.21 \pm 0.12^{* *}(25.14)$ & $14.37 \pm 0.03^{* *}(40.51)$ \\
S15 & $6.97 \pm 0.07^{* *}(3.33)$ & $10.16 \pm 0.2^{* *}(33.59)$ & &
\end{tabular}


(i) Continued.

\begin{tabular}{|c|c|c|c|c|}
\hline \multirow{2}{*}{ Complexes } & \multicolumn{4}{|c|}{ Concentrations $\left(\mu \mathrm{gmL}^{-1}\right)$} \\
\hline & 5 & 10 & 20 & 40 \\
\hline $\operatorname{ANOVA}(\mathrm{df}=10,22)$ & F-1304.177 $P<0.001$ & F-1543.733 $P<0.001$ & F-622.545 $P<0.001$ & F-420.443P $<0.001$ \\
\hline \multicolumn{5}{|c|}{ Mean \pm S.D (percent zone of inhibition), ${ }^{* *} P<0.005,{ }^{*} P<0.05$} \\
\hline \multicolumn{5}{|c|}{ (j) } \\
\hline \multirow{2}{*}{ Complexes } & \multicolumn{4}{|c|}{ Concentrations $\left(\mu \mathrm{gmL}^{-1}\right)$} \\
\hline & 5 & 10 & 20 & 40 \\
\hline SPFX & $7.21 \pm 0.2$ & $15.3 \pm 0.21$ & $16.31 \pm 0.04$ & $17.28 \pm 0.11$ \\
\hline S12 & $8.09 \pm 0.36^{* *}(-12.21)$ & $9.29 \pm 0.1^{* *}(39.28)$ & $11.1 \pm 0.08^{* *}(31.94)$ & $14.05 \pm 0.25^{* *}(18.69)$ \\
\hline S13 & $8.84 \pm 0.16^{* *}(-22.61)$ & $11.01 \pm 0.27^{* *}(28.04)$ & $15.08 \pm 0.18^{* *}(7.54)$ & $18.32 \pm 0.14^{* *}(-6.02)$ \\
\hline S14 & $0 \pm 0^{* *}(100)$ & $0 \pm 0^{* *}(100)$ & $9.31 \pm 0.25^{* *}(42.92)$ & $10.28 \pm 0.23^{* *}(40.51)$ \\
\hline S15 & $6.97 \pm 0.07^{* *}(3.33)$ & $10.16 \pm 0.2^{* *}(33.59)$ & $12.21 \pm 0.12^{* *}(25.14)$ & $14.37 \pm 0.06^{* *}(16.84)$ \\
\hline ANOVA $(\mathrm{df}=10,22)$ & F-1304.177 $P<0.001$ & F-1543.733 $P<0.001$ & F-622.545 $P<0.001$ & F-420.443 $P<0.001$ \\
\hline
\end{tabular}

Mean \pm S.D (percent zone of inhibition), ${ }^{* *} P<0.005,{ }^{*} P<0.05$.

(k)

\begin{tabular}{|c|c|c|c|c|}
\hline \multirow{2}{*}{ Complexes } & \multicolumn{4}{|c|}{ Concentrations $\left(\mu \mathrm{gmL}^{-1}\right)$} \\
\hline & 5 & 10 & 20 & 40 \\
\hline SPFX & $7.21 \pm 0.2$ & $15.3 \pm 0.21$ & $16.31 \pm 0.04$ & $17.28 \pm 0.11$ \\
\hline S12 & $8.09 \pm 0.36^{* *}(-12.21)$ & $9.29 \pm 0.1^{* *}(39.28)$ & $11.1 \pm 0.08^{* *}(31.94)$ & $14.05 \pm 0.25^{* *}(18.69)$ \\
\hline S13 & $8.84 \pm 0.16^{* *}(-22.61)$ & $11.01 \pm 0.27^{* *}(28.04)$ & $15.08 \pm 0.18^{* *}(7.54)$ & $18.32 \pm 0.14^{* *}(-6.02)$ \\
\hline S14 & $0 \pm 0^{* *}(100)$ & $0 \pm 0^{* *}(100)$ & $9.31 \pm 0.25^{* *}(42.92)$ & $10.28 \pm 0.23^{* *}(40.51)$ \\
\hline S15 & $6.97 \pm 0.07^{* *}(3.33)$ & $10.16 \pm 0.2^{* *}(33.59)$ & $12.21 \pm 0.12^{* *}(25.14)$ & $14.37 \pm 0.06^{* *}(16.84)$ \\
\hline $\operatorname{ANOVA}(\mathrm{df}=10,22)$ & F-1304.177 $P<0.001$ & F-1543.733 $P<0.001$ & F-622.545 $P<0.001$ & F-420.443 $P<0.001$ \\
\hline
\end{tabular}

Mean \pm S.D (percent zone of inhibition), ${ }^{* *} P<0.005,{ }^{*} P<0.05$.

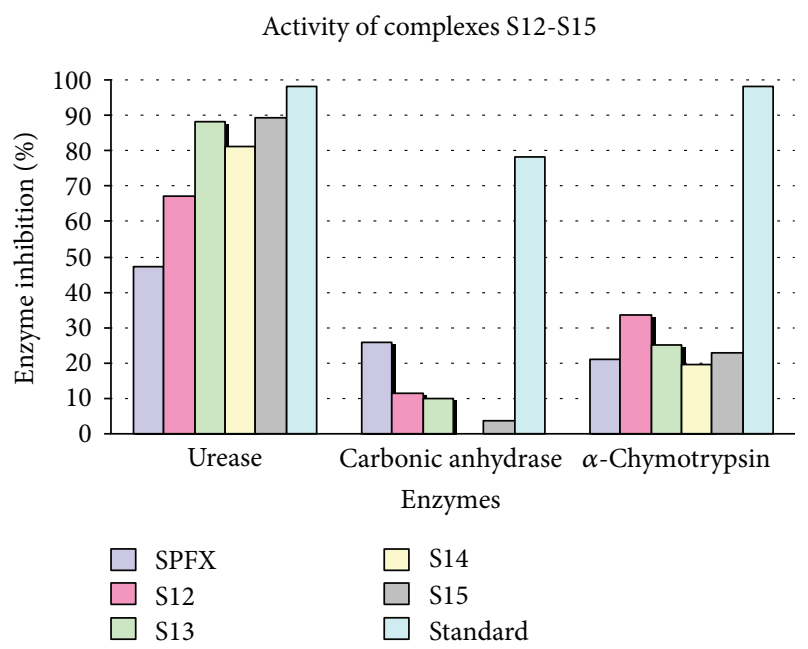

FIGURE 4: Graphical representation of enzymatic inhibition S12 to S15.

\subsection{Antimicrobial Studies}

3.4.1. Antibacterial Activity. Comparison of antibacterial activity data of novel SPFX metal complexes suggests that almost all complexes are active antimicrobial agents (Tables 5(a)-5(k), Figure 2) and most of them exhibit better activity than parent drug. SPFX complexes including S13, S14, and S15 were found to be the most active complexes possessing higher antimicrobial activity against $B$. subtilis and M. luteus in all four tested concentrations. All synthesized complexes show moderate activity against $S$. aureus and $S$. features as compared to parent drug as well as other advance fluoroquinolones. Spectrum of Gram-negative activity indicated that all complexes show remarkable (excellent) activity against $P$. aeruginosa, E.coli, and S. typhe while S13, S14, and S15 exhibit good activity against $P$. mirabilis and citrobacter in comparison to SPFX and other standards, M. luteus and S. typhe. All complexes show almost same or less activity against K. pneumoniae and S. flexneri.

3.4.2. Antifungal Activity. These synthesized complexes were also evaluated for antifungal activity. The average results are shown in Tables 6(a)-6(c) (Figure 3). All of the complexes show excellent activity against $F$. solani as compared to the parent drug and other standards, while all synthesized complexes are less active against T. rubrum and more or less equally potent against C. albican in comparison to parentdrug. None of complexes show activity against $A$. parasiticus, $A$. effuris, and $S$. cervicis. In general, antifungal data and its statistical analysis confirm that all four synthesized complexes are most active and show significant activity against $F$. solani with respect to parent drug as well as other advance fluoroquinolones. 
TABle 6: (a) Inhibition zones (mm) against C. albicans. (b) Inhibition zones (mm) against F. solani. (c) Inhibition zones (mm) against T. rubrum.

(a)

\begin{tabular}{lcccc}
\hline Complexes & 5 & \multicolumn{3}{c}{ Concentrations $\left(\mu \mathrm{gmL}^{-1}\right)$} \\
& 5 & 10 & $16.31 \pm 0.04$ & 40 \\
\hline SPFX & $7.21 \pm 0.2$ & $15.3 \pm 0.21$ & $17.28 \pm 0.11$ \\
S12 & $8.09 \pm 0.36^{* *}(-12.21)$ & $9.29 \pm 0.1^{* *}(39.28)$ & $11.08^{* *}(31.94)$ & $14.05 \pm 0.25^{* *}(18.69)$ \\
S13 & $8.84 \pm 0.16^{* *}(-22.61)$ & $11.01 \pm 0.27^{* *}(28.04)$ & $15.08 \pm 0.18^{* *}(7.54)$ & $18.32 \pm 0.14^{* *}(-6.02)$ \\
S14 & $0 \pm 0^{* *}(100)$ & $0 \pm 0^{* *}(100)$ & $9.31 \pm 0.25^{* *}(42.92)$ & $10.28 \pm 0.23^{* *}(40.51)$ \\
S15 & $6.97 \pm 0.07^{* *}(3.33)$ & $10.16 \pm 0.2^{* *}(33.59)$ & $12.21 \pm 0.12^{* *}(25.14)$ & $14.37 \pm 0.06^{* *}(16.84)$ \\
ANOVA $(\mathrm{df}=10,22)$ & $\mathrm{F}-270.407 P<0.001$ & $\mathrm{~F}-26.145 P<0.001$ & $\mathrm{~F}-78.461 P<0.001$ & $\mathrm{~F}-52.516 P<0.001$ \\
\hline
\end{tabular}

Mean \pm S.D (percent zone of inhibition), ${ }^{* *} P<0.005,{ }^{*} P<0.05$.

(b)

\begin{tabular}{lcccc}
\hline Complexes & \multicolumn{3}{c}{ Concentrations $\left(\mu \mathrm{gmL}^{-1}\right)$} \\
& 5 & 10 & 20 & 40 \\
\hline SPFX & $0 \pm 0$ & $0 \pm 0$ & $10.2 \pm 0.04$ & $11.12 \pm 0.07$ \\
S12 & $20.23 \pm 0.24^{* *}(0)$ & $22.2 \pm 0.22^{* *}(0)$ & $23.29 \pm 0.06^{* *}(-128.33)$ & $27.06 \pm 0.2^{* *}(-143.35)$ \\
S13 & $22.06 \pm 0.06^{* *}(0)$ & $24.06 \pm 0.15^{* *}(0)$ & $28.22 \pm 0.09^{* *}(-176.67)$ & $30.04 \pm 0.02^{* *}(-170.14)$ \\
S14 & $12.09 \pm 0.22^{* *}(0)$ & $15.98 \pm 0.04^{* *}(0)$ & $21.17 \pm 0.35^{* *}(-107.55)$ & $24.24 \pm 0.06^{* *}(-117.99)$ \\
S15 & $17.26 \pm 0.14^{* *}(0)$ & $22.03 \pm 0.27^{* *}(0)$ & $26.08 \pm 0.16^{* *}(-155.69)$ & $30.13 \pm 0.2^{* *}(-170.95)$ \\
ANOVA $(\mathrm{df}=10,22)$ & $\mathrm{F}-67.16 P<0.001$ & $\mathrm{~F}-124.599 P<0.001$ & $\mathrm{~F}-246.703 P<0.001$ & $\mathrm{~F}-133.773 P<0.001$ \\
\hline
\end{tabular}

Mean \pm S.D (percent zone of inhibition), ${ }^{* *} P<0.005,{ }^{*} P<0.05$.

(c)

\begin{tabular}{lcccc}
\hline Complexes & \multicolumn{4}{c}{ Concentrations $\left(\mu \mathrm{gmL}^{-1}\right)$} \\
& 5 & 10 & 20 & 40 \\
\hline SPFX & $0 \pm 0$ & $20.4 \pm 0.07$ & $23.07 \pm 0.05$ & $24.29 \pm 0.13$ \\
S12 & $22.22 \pm 0.14^{* *}(0)$ & $22.07 \pm 0.4^{* *}(-8.19)$ & $24.24 \pm 0.15^{* *}(-5.07)$ & $27.06 \pm 0.09^{* *}(-11.4)$ \\
S13 & $18.95 \pm 0.37^{* *}(0)$ & $25.31 \pm 0.07^{* *}(-24.07)$ & $26.27 \pm 0.27^{* *}(-13.87)$ & $27.08 \pm 0.31^{* *}(-11.49)$ \\
S14 & $12.22 \pm 0.09^{* *}(0)$ & $17.02 \pm 0.17^{* *}(16.57)$ & $19.11 \pm 0.08^{* *}(17.17)$ & $22.18 \pm 0.07^{* *}(8.69)$ \\
S15 & $18.24 \pm 0.1^{* *}(0)$ & $21.32 \pm 0.17^{* *}(-4.51)$ & $24.1 \pm 0.03^{* *}(-4.46)$ & $27.25 \pm 0.19^{* *}(-12.19)$ \\
ANOVA (df $=10,22)$ & $\mathrm{F}-252.324 P<0.001$ & $\mathrm{~F}-2663.194 P<0.001$ & $\mathrm{~F}-476.634 P<0.001$ & $\mathrm{~F}-315.709 P<0.001$ \\
\hline
\end{tabular}

Mean \pm S.D (percent zone of inhibition), ${ }^{* *} P<0.005,{ }^{*} P<0.05$.

TABLE 7: Enzymatic activities of SPFX metal complexes.

\begin{tabular}{|c|c|c|c|c|c|c|}
\hline \multirow{2}{*}{$\begin{array}{l}\text { Enzymes } \\
\text { Compounds }\end{array}$} & \multicolumn{2}{|c|}{ Urease } & \multicolumn{2}{|c|}{ Carbonic anhydrase } & \multicolumn{2}{|c|}{$\alpha$-Chymotrypsin } \\
\hline & Inhibition (\%) & $\mathrm{IC}_{50} \pm \operatorname{SEM}(\mu \mathrm{m})$ & Inhibition (\%) & $\mathrm{IC}_{50} \pm \operatorname{SEM}(\mu \mathrm{m})$ & Inhibition (\%) & $\mathrm{IC}_{50} \pm \operatorname{SEM}(\mu \mathrm{m})$ \\
\hline SPAR & 47.3 & - & 26.00 & - & 21.1 & - \\
\hline S12 & 67.20 & $128.53 \pm 0.11$ & 11.30 & - & 33.5 & - \\
\hline S13 & 88.30 & $316.2 \pm 0.60$ & 10.00 & - & 25.1 & - \\
\hline S14 & 81.20 & $169.96 \pm 0.26$ & - & - & 19.6 & - \\
\hline S15 & 89.20 & $145.13 \pm 0.09$ & 3.70 & - & 22.9 & - \\
\hline Standard & 98.2 & $21.00 \pm 0.11$ & 78.1 & $0.30 \pm 0.0006$ & 98.1 & $5.70 \pm 0.13$ \\
\hline
\end{tabular}

3.5. Enzymatic Activity. To study inhibitory effects of newly formed complexes, enzyme inhibition studies have been conducted against urease, $\alpha$-Chymotrypsin, and carbonic anhydrase (Table 7, Figure 4). Results indicated that all complexes exhibit very good activities against urease as compared to standard (thiourea), while all complexes show no or little activity against carbonic anhydrase using acetazolamide as reference standard.

\section{Conclusion}

Metal complexes of SPFX via heavy metal have been synthesized in good yield and characterized by physicochemical and spectroscopic methods. Sparfloxacinato ligand binds with metals through pyridine and oxygen atom of carboxylic group. The biological activities of complexes have been tested against various bacteria and fungi; it was observed that S13, 
S14, and S15 were found to be most active complexes and possess higher antimicrobial activity against $B$. subtilis and $M$. luteus in all four tested concentrations but less active than the parent drug, while all complexes show almost same or less activity against $K$. pneumoniae and $S$. flexneri. Antifungal data confirm that all four synthesized complexes are most active and show significant activity against F.solani with respect to parent drug as well as other advance fluoroquinolones and none of complexes show activity against $A$. parasiticus, $A$. effuris, and S. cervicis. Enzymatic activity results of these complexes indicated them to be good inhibitors of urease enzyme while all complexes show mild activities against carbonic anhydrase enzyme. Further research may prove promising role of these synthesized complexes as urease inhibitors.

\section{References}

[1] J. Kang, L. Wang, X.-L. Chen, D. J. Triggle, and D. Rampe, "Interactions of a series of fluoroquinolone antibacterial drugs with the human cardiac K+ channel HERG," Molecular Pharmacology, vol. 59, no. 1, pp. 122-126, 2001.

[2] W. Mertz, "Review of the scientific basis for establishing the essentiality of trace elements," Biological Trace Element Research, vol. 66, no. 1-3, pp. 185-191, 1998.

[3] E. K. Efthimiadou, Y. Sanakis, N. Katsaros, A. Karaliota, and G. Psomas, "Transition metal complexes with the quinolone antibacterial agent pipemidic acid: synthesis, characterization and biological activity," Polyhedron, vol. 26, no. 5, pp. 1148-1158, 2007.

[4] M. S. Arayne, N. Sultana, and F. Hussain, "In vitro monitoring of ciprofloxacin antacids interactions by UV \& HPLC," Pakistan Journal of Pharmaceutical Sciences, vol. 18, no. 4, pp. 23-31, 2005.

[5] J. Al-Mustafa, "Magnesium, calcium and barium perchlorate complexes of ciprofloxacin and norfloxacin," Acta Chimica Slovenica, vol. 49, no. 3, pp. 457-466, 2002.

[6] S. Sagdinca and S. Bayarýb, "Spectroscopic studies on the interaction of ofloxacin with metals," Journal of Molecular Structure, vol. 691, no. 1-3, pp. 107-113, 2004.

[7] I. Turel, "The interactions of metal ions with quinolone antibacterial agents," Coordination Chemistry Reviews, vol. 232, no. 1-2, pp. 27-47, 2002.

[8] B. Macías, M. V. Villa, I. Rubio, A. Castiñeiras, and J. Borrás, "Complexes of $\mathrm{Ni}(\mathrm{II})$ and $\mathrm{Cu}(\mathrm{II})$ with ofloxacin: crystal structure of a new $\mathrm{Cu}(\mathrm{II})$ ofloxacin complex," Journal of Inorganic Biochemistry, vol. 84, no. 3-4, pp. 163-170, 2001.

[9] N. Sultana, M. S. Arayne, S. Gul, and S. Shamim, "Sparfloxacinmetal complexes as antifungal agents-their synthesis, characterization and antimicrobial activities," Journal of Molecular Structure, vol. 975, no. 1-3, pp. 285-291, 2010.

[10] G. Hoffken, K. Borner, P. D. Glatzel, P. Koeppe, and H. Lode, "Reduced enteral absorption of ciprofloxacin in the presence of antacids," European Journal of Clinical Microbiology, vol. 4, no. 3, p. 345, 1985.

[11] H. H. M. Ma, F. C. K. Chiu, and R. C. Li, "Mechanistic investigation of the reduction in antimicrobial activity of ciprofloxacin by metal cations," Pharmaceutical Research, vol. 14, no. 3, pp. 366-370, 1997.

[12] H. N. Alkaysi, M. H. Abdel-Hay, M. Sheikh Salem, A. M. Gharaibeh, and T. E. Na'was, "Chemical and microbiological investigations of metal ion interaction with norfloxacin," International Journal of Pharmaceutics, vol. 87, no. 1-3, pp. 73-77, 1992.

[13] E. K. Efthimiadou, A. Karaliota, and G. Psomas, "Mononuclear dioxomolybdenum(VI) complexes with the quinolones enrofloxacin and sparfloxacin: synthesis, structure, antibacterial activity and interaction with DNA," Polyhedron, vol. 27, no. 1, pp. 349-356, 2008.

[14] E. K. Efthimiadou, Y. Sanakis, N. Katsaros, A. Karaliota, and G. Psomas, "Transition metal complexes with the quinolone antibacterial agent pipemidic acid: synthesis, characterization and biological activity," Polyhedron, vol. 26, no. 5, pp. 1148-1158, 2007.

[15] E. K. Efthimiadou, Y. Sanakis, C. P. Raptopoulou, A. Karaliota, N. Katsaros, and G. Psomas, "Crystal structure, spectroscopic, and biological study of the copper(II) complex with thirdgeneration quinolone antibiotic sparfloxacin," Bioorganic and Medicinal Chemistry Letters, vol. 16, no. 14, pp. 3864-3867, 2006.

[16] E. K. Efthimiadou, M. E. Katsarou, A. Karaliota, and G. Psomas, "Copper(II) complexes with sparfloxacin and nitrogen-donor heterocyclic ligands: structure-activity relationship," Journal of Inorganic Biochemistry, vol. 102, no. 4, pp. 910-920, 2008.

[17] K. C. Skyrianou, E. K. Efthimiadou, V. Psycharis, A. Terzis, D. P. Kessissoglou, and G. Psomas, "Nickel-quinolones interactionpart 1: nickel(II) complexes with the antibacterial drug sparfloxacin: structure and biological properties," Journal of Inorganic Biochemistry, vol. 103, no. 12, pp. 1617-1625, 2009.

[18] G. Psomas, "Mononuclear metal complexes with ciprofloxacin: synthesis, characterization and DNA-binding properties," Journal of Inorganic Biochemistry, vol. 102, no. 9, pp. 1798-1811, 2008.

[19] H. N. Alkaysi, M. H. Abdel-Hay, M. Sheikh Salem, A. M. Gharaibeh, and T. E. Na'was, "Chemical and microbiological investigations of metal ion interaction with norfloxacin," International Journal of Pharmaceutics, vol. 87, no. 1-3, pp. 73-77, 1992.

[20] I. Turel, "The interactions of metal ions with quinolone antibacterial agents," Coordination Chemistry Reviews, vol. 232, no. 1-2, pp. 27-47, 2002.

[21] I. Turel, I. Leban, and N. Bukovec, "Synthesis, characterization, and crystal structure of a copper(II) complex with quinolone family member (ciprofloxacin): bis(1-cyclopropyl-6-fluoro- 1,4dihydro-4-oxo-7-piperazin-1ylquinoline-3-carboxylate) copper (II) chloride hexahydrate," Journal of Inorganic Biochemistry, vol. 56, no. 4, pp. 273-282, 1994.

[22] I. Turel, K. Gruber, I. Leban, and N. Bukovec, "Synthesis, crystal structure, and characterization of three novel compounds of the quinolone family member (norfloxacin)," Journal of Inorganic Biochemistry, vol. 61, no. 3, pp. 197-212, 1996.

[23] S. C. Wallis, L. R. Gahan, B. G. Charles, and T. W. Hambley, "Synthesis and X-ray structural characterization of an iron(III) complex of the fluoroquinolone antimicrobial ciprofloxacin," Polyhedron, vol. 14, no. 20-21, pp. 2835-2840, 1995.

[24] S. C. Wallis, L. R. Gahan, B. G. Charles, T. W. Hambley, and P. A. Duckworth, "Copper(II) complexes of the fluoroquinolone antimicrobial ciprofloxacin. Synthesis, X-ray structural characterization, and potentiometric study," Journal of Inorganic Biochemistry, vol. 62, no. 1, pp. 1-16, 1996.

[25] Z.-F. Chen, R.-G. Xiong, J. Zhang, X.-T. Chen, Z.-L. Xue, and X.Z. You, "2D molecular square grid with strong blue fluorescent emission: a complex of norfloxacin with zinc(II)," Inorganic Chemistry, vol. 40, no. 16, pp. 4075-4077, 2001. 
[26] B. Macías, M. V. Villa, I. Rubio, A. Castiñeiras, and J. Borrás, "Complexes of $\mathrm{Ni}(\mathrm{II})$ and $\mathrm{Cu}(\mathrm{II})$ with ofloxacin: crystal structure of a new $\mathrm{Cu}(\mathrm{II})$ ofloxacin complex," Journal of Inorganic Biochemistry, vol. 84, no. 3-4, pp. 163-170, 2001.

[27] L.-Z. Wang, Z.-F. Chen, X.-S. Wang, Y.-H. Li, R.-G. Xiong, and X.-Z. You, "2D nanoporous molecular square grid: manganese (II) norfloxacin complex," Chinese Journal of Inorganic Chemistry, vol. 18, no. 12, pp. 1185-1190, 2002.

[28] M. S. Arayne, N. Sultana, and F. Hussain, "Interactions between sparfloxacin and antacids: dissolution and adsorption studies," Pakistan Journal of Pharmaceutical Sciences, vol. 19, no. 1, pp. 16-21, 2006.

[29] S. Arayne, N. Sultana, U. Haroon, and M. A. Mesaik, "Synthesis, characterization, antibacterial and anti-inflammatory activities of enoxacin metal complexes," Bioinorganic Chemistry and Applications, vol. 2009, Article ID 914105, 6 pages, 2009.

[30] N. Sultana, A. Naz, M. S. Arayne, and M. A. Mesaik, "Synthesis, characterization, antibacterial, antifungal and immunomodulating activities of gatifloxacin-metal complexes," Journal of Molecular Structure, vol. 969, no. 1-3, pp. 17-24, 2010.

[31] A. W. Bauer, W. M. Kirby, J. C. Sherris, and M. Turck, "Antibiotic susceptibility testing by a standardized single disk method," The American Journal of Clinical Pathology, vol. 45, no. 4, pp. 493496, 1966.

[32] National Committee on Clinical Laboratory Standards, Performance Standards for Antimicrobial Disk Susceptibility Tests, NCCLS, Villanova, Pa, USA, 1983.

[33] LMD Victor Antibiotics in Laboratory Medicine, Williams and Wilkins, 3rd edition, 1991.

[34] E. K. Efthimiadou, A. Karaliota, and G. Psomas, "Structure, antimicrobial activity and DNA-binding properties of the cobalt(II)-sparfloxacin complex," Bioorganic and Medicinal Chemistry Letters, vol. 18, no. 14, pp. 4033-4037, 2008.

[35] E. K. Efthimiadou, Y. Sanakis, C. P. Raptopoulou, A. Karaliota, N. Katsaros, and G. Psomas, "Crystal structure, spectroscopic, and biological study of the copper(II) complex with thirdgeneration quinolone antibiotic sparfloxacin," Bioorganic and Medicinal Chemistry Letters, vol. 16, no. 14, pp. 3864-3867, 2006.

[36] E. K. Efthimiadou, M. E. Katsarou, A. Karaliota, and G. Psomas, "Copper(II) complexes with sparfloxacin and nitrogen-donor heterocyclic ligands: structure-activity relationship," Journal of Inorganic Biochemistry, vol. 102, no. 4, pp. 910-920, 2008.

[37] S. Jain, N. K. Jain, and K. S. Pitre, "Bio-inorganic studies on the Fe(II) sparfloxacin complex," Metal-Based Drugs, vol. 9, no. 1-2, pp. 1-8, 2002.

[38] E. Prestch, T. Clerc, J. Seibl, and W. Simon, Tablas de Determinacion Estructural Por Metodos Espectroscopicos, Springer, Barcelona, Spain, 1998.

[39] N. Jiménez-Garrido, L. Perelló, R. Ortiz et al., “Antibacterial studies, DNA oxidative cleavage, and crystal structures of $\mathrm{Cu}(\mathrm{II})$ and $\mathrm{Co}$ (II) complexes with two quinolone family members, ciprofloxacin and enoxacin," Journal of Inorganic Biochemistry, vol. 99, no. 3, pp. 677-689, 2005.

[40] M. Saeed Arayne, N. Sultana, and A. Zeeshan Mirza, "Preparation and spectroscopic characterization of metal complexes of gliquidone," Journal of Molecular Structure, vol. 927, no. 1-3, pp. 54-59, 2009. 

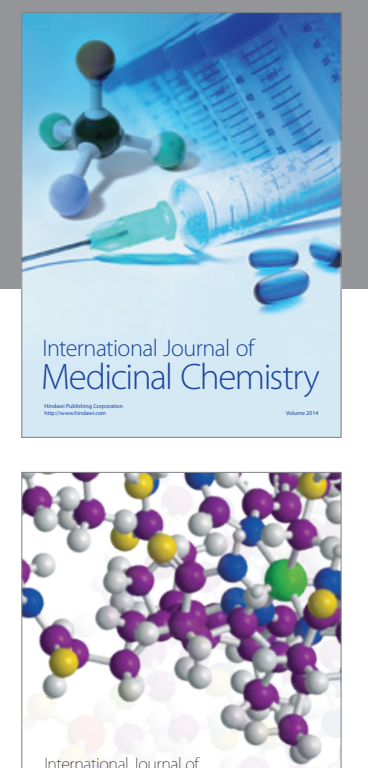

\section{Carbohydrate} Chemistry

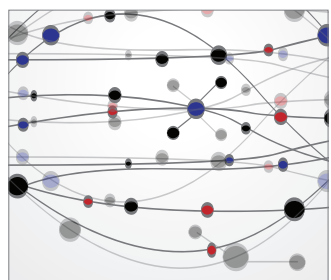

The Scientific World Journal
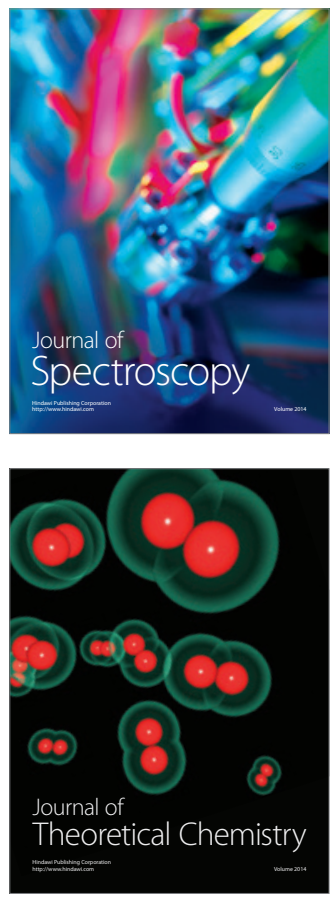
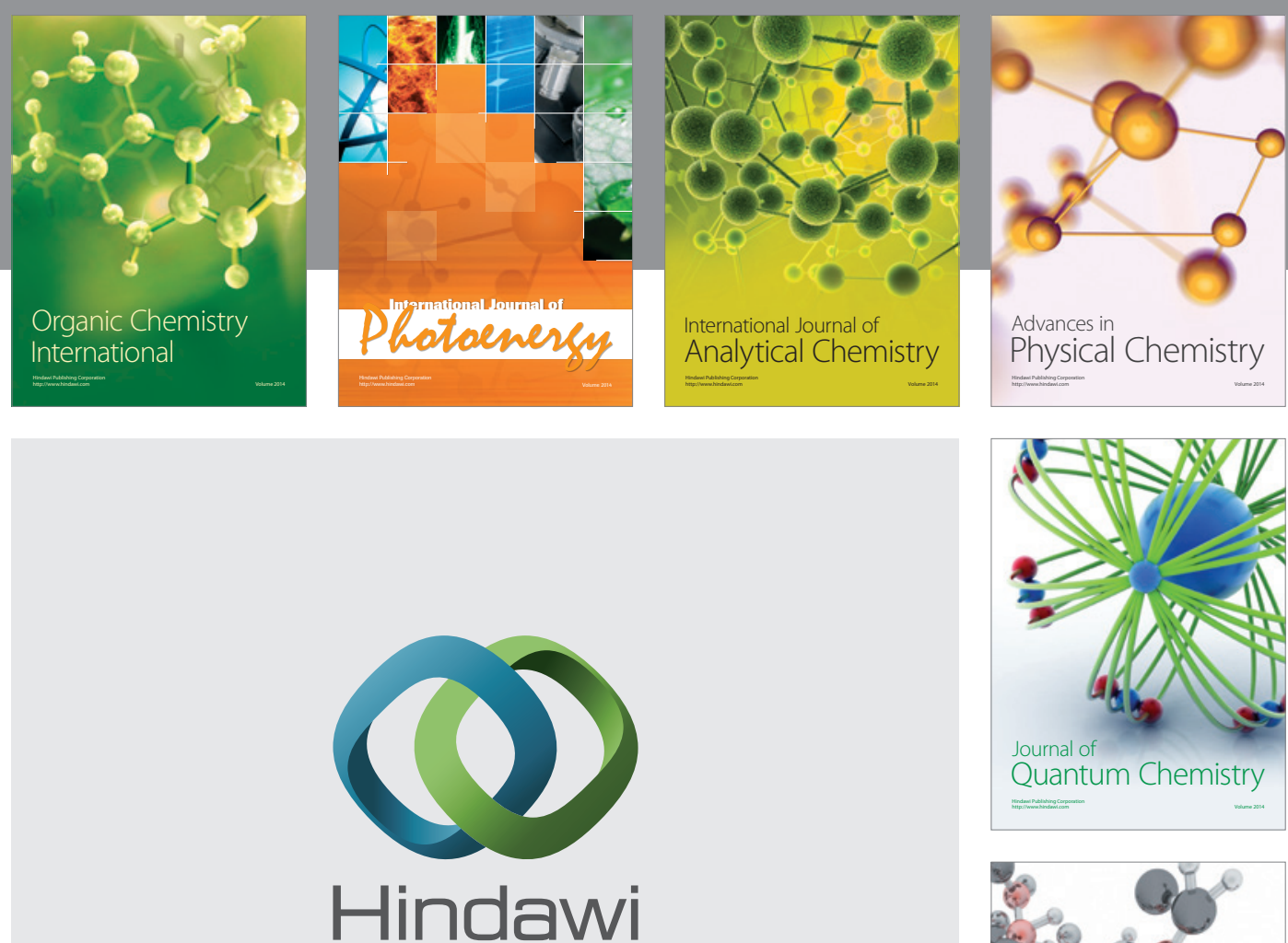

Submit your manuscripts at

http://www.hindawi.com

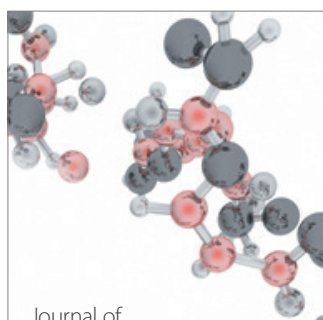

Analytical Methods

in Chemistry

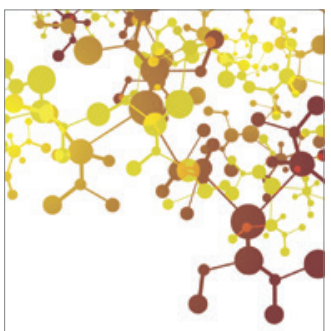

Journal of

Applied Chemistry

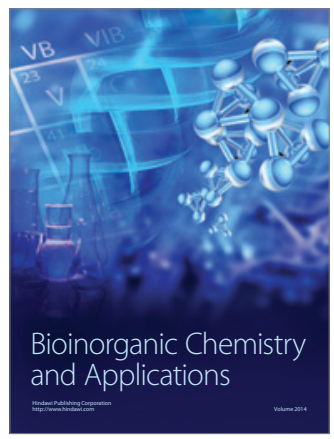

Inorganic Chemistry
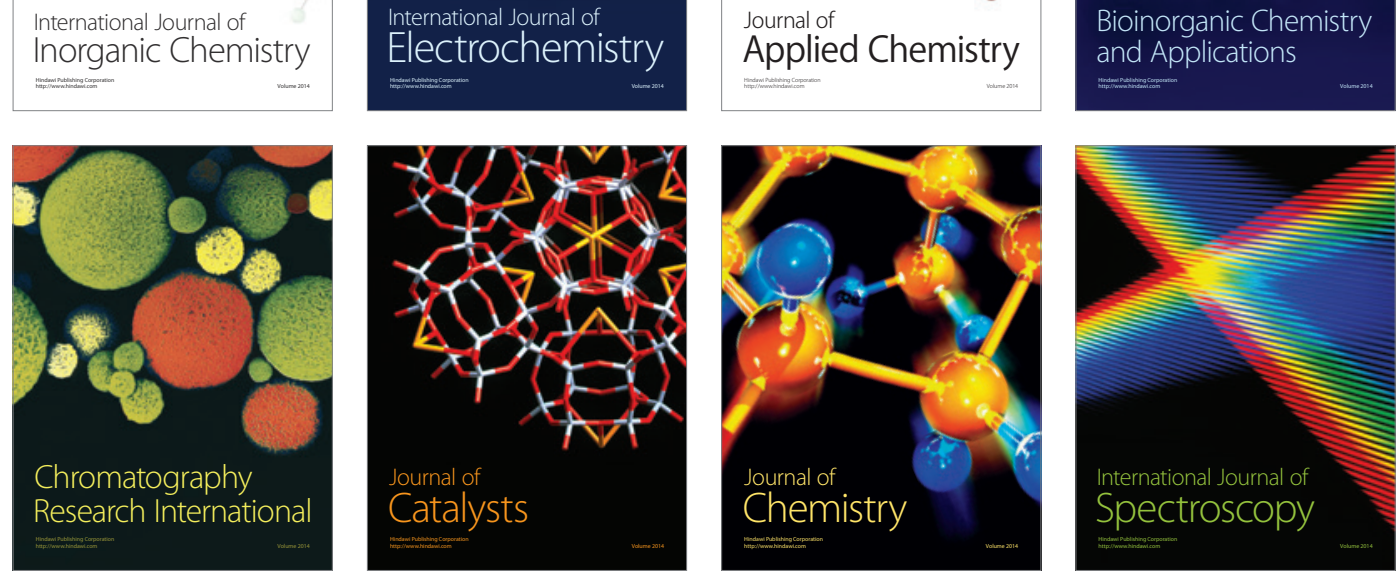